\title{
Knowledge and social networks: New dimensions of economic interaction between firms
}

\author{
Michael Steiner * and Michael Ploder ** \\ * University of Graz, Department of Economics, Universitätsstr. 15 F/4, 8010 Graz, Austria \\ ** Joanneum Research, Institute of Technology and Regional Policy, Elisabethstr. 20, 8010 \\ Graz, Austria \\ michael.steiner@uni-graz.at \\ michael.ploder@joanneum.at
}

Received 20 August 2011; received in revised form 20 November 2011; accepted 29 December 2011

\begin{abstract}
The paper explores the form and content of economic interaction of firms based on various concepts of agglomeration and social networks. It uses a case study of the machinery sector in the region of Styria as empirical background. Starting with types of clustering - the model of pure agglomeration, the industrial-complex model and the social-network model - the paper argues that certain geographical agglomerations allow different types of networks and different patterns of behaviour. Thus different forms of learning, knowledge sharing and knowledge creation. Some "stylized facts" in support of this perspective are derived from an analysis of a regional network. This network comprises individualistic open systems consisting of several areas which overlap. Physical linkages between these networks are weak, but intersections based on cooperative $\mathrm{R} \& \mathrm{D}$ and $\mathrm{R} \& \mathrm{D}$ infrastructure, qualification and informal exchanges are evident. From a regional perspective it can be seen to dominate. Despite evident sectoral concentrations direct links to the prevailing science base appear more significant as binding factors than long term supplier networks. These relationships are interpreted in terms of their need for proximity, their durability and above all their direction of knowledge dependency.
\end{abstract}

Keywords: Agglomeration, Knowledge Transfer, Social Networks, co-evolutionary development 


\section{Introduction}

While we are well aware that it is most likely impossible to provide one single theory of clusters and their networks there is nevertheless a certain consensus that several elements of specific theories may help us understand their forms and functions. They offer a certain unity of approach in identifying the important elements which are needed for explaining the changing character of the innovation process.

Recent debate has begun to focus more on how far, and in which ways, clusters foster knowledge creation and organizational learning. It has also emphasized the organic-evolutionary dimension of cluster-based industrial agglomerations. Knowledge has been recognized as a major source of competitive advantage in an increasingly integrated world economy (Dosi and Malerba 1996, Grant 1996, Foss 1999, Nonaka et al. 2000). The most successful regions are perceived to be those whose firms display innovative capacity, i. e. those whose firms are able to adapt to a rapidly changing marketplace and stay one step ahead of competitors. The emphasis of cluster interpretation has changed from an analysis of forces of agglomeration to the various forms and contents of organizational learning and knowledge exchange. The original concentration on clusters as mere geographic concentrations of sectors and firms has been transformed into a search for institutions for knowledge management and organizational learning emphasizing the organic-evolutionary dimension. Growth of the knowledge base depends on intended and unintended individual processing of experiences. I.e. 'learning', while the interpretation, transfer and use of experiences is influenced by interaction between individuals and between organizations (Cohen and Levinthal 1989, Andersen 1995, Hartmann 2006). These insights have shifted the emphasis from material links to immaterial knowledge flows within clusters. They have also pointed to the need for connectivity between different agents concerning knowledge creation and diffusion. This has led to further questions concerning the degree to which clusters are to be regarded as non-market devices, by which firms may seek to coordinate their activities with other firms and knowledge-generating institutions. Ongoing learning processes between firms and within clusters stress the importance of institutional arrangements for the generation of knowledge and learning networks which are not available in markets (Maskell and Malmberg 1999). Since the necessary knowledge may lie outside a firm's traditional core competence, interfirm alliances and networks are widely recognized as an important organization form of innovative activity (Gay and Dousset 2005).
In this paper, we emphasize the ideas of agglomeration and knowledge exchange.We discuss to what extent this approach has specific regional or spatial dimensions while focussing on the necessity and forms of proximity, especially with respect to knowledge exchange.

By means of network analysis we develop some "stylized facts" for the various dimensions of interaction within a given network of medium-tech firms in Styria, one of the nine provinces (regions) of Austria. The final section is used to interpret the findings.

\section{Geographical agglomeration and local networks}

Since Marshall (1890), Weber (1929) and Hoover (1948), many authors have dealt with the phenomenon of geographical agglomeration. In the discussions of 'clusters', 'networks' and agglomerations, and particularly in those relating to 'industrial districts' and agglomerations, there are certain common traits and frequently terms are only weakly differentiated. The basic idea of geographical agglomeration was presented by Marshall (1890) and the three sources of economies of agglomeration he mention is input sharing, labor market pooling, and knowledge spillovers, correspond with the core-elements of the current cluster-concept. In this form it has been discussed since the early nineties in industrial countries. A more recent attempt to distinguish various cluster forms has been made by Belussi (2006) by contrasting geographical agglomeration and active clustering (as policy or firm-driven strategy).

While implicitly focusing on geographical agglomeration and economies of agglomeration we stress a dimension of externalities beyond the tangible dimension of direct co-operation. On extending the basic idea of economies of agglomeration, we see that externalities are widely enforced by informal and non-economic dimensions. Amin and Thrift (1995) use the term "institutional thickness" to address the existence of a supporting environment beyond firms (institutionalized cooperations and networks). Geographic agglomeration (and concentrated versus dispersed location patterns) set a framework for economic interaction and material and immaterial linkages between economic actors.

The existence of a cluster doesn't necessarily imply the coexistence of all defining characteristics of a geographical agglomeration. On the other hand, a geographical agglomeration may also exist in the absence of a cluster or network.

While the existence of a pure geographical agglomeration (e.g. a city) favours the development of clusters; growing networks and clusters can also cause the emergence of a geographical 
agglomeration. This was the case perhaps in Silicon Valley in California. Myrdal's (1957) idea of cumulative causation corresponds with a dynamic view of a co-evolutionary development of economies of agglomeration and growing clusters (without yet formalizing interdependency as was done by Kaldor (1972) and Dixon and Thirlwall (1975). In other words, additional local linkages and relations strengthen tendencies of concentration and agglomeration. Networks and clusters are possible means of overcoming constraints of exchange within and between geographical agglomerations and also facilitate the definition and defence of rules of exclusion, as already pointed out by Marshall (1890). Yet, what is still an open question is the micro-perspective. Economies of agglomeration and dimensions of interaction could be selective in respect to the actors since they regulate the extent to which the latter are able to participate or gain from externalities: e.g. with respect to exchange of physical goods versus $R \& D$, or labour market pools for blue collar workers or engineers.

In addition to direct physical exchange, input sharing and common labour market pools, systematic knowledge exchange and knowledge spillovers have gained considerably in importance as an argument for geographical concentrations of activities. A frequently used argument is that the collaborative nature of innovation processes has reinforced tendencies toward geographical clustering because of the advantages of locating in close proximity to other firms in specialized and related industries (Storper, 1995 and 1997). Transaction costs such as transportation costs and spatial communication costs in particular, reinforce the relationship between individual environment and the development of embedded social networks (Granovetter 1994).

Firms establish a variety of types of interactions and relationships each of them having different impacts on the knowledge generation and diffusion process. Mariotti and Delbridge (2001) speak of the necessity for firms - in the face of knowledge ambiguity, knowledge related barriers, tacitness and complexity of knowledge to engage in the management of a portfolio of ties. Organizations are therefore likely to engage in inter-organizational relations that show a variety of types of ties.

They can have quite different dimensions and can be defined according to the character of social relations between actors, the regulation of the relationship, frequency of use, length and duration of the relationship, and also in terms of the nature of the information exchange itself (Mariotti and Delbridge 2001). It is also important to distinguish between both content (i.e. the type of relation) and the form (i.e. the social structure of relations), as has been outlined by Powell and Smith-Doerr (1994).
One additional question that needs to be addressed in this context concerns the legitimacy of a pure micro-level, individual firm approach in analysing the incentives for clustering.

Individuals and firms alone are, from an economic point of view, not capable of delivering sufficient amounts and varieties of knowledge. We are confronted here with one of "the most troublesome issues in the social sciences ..." (Felin and Foss 2006, 1). The question of the adequate level and unit of analysis. A question of whether the individual or social collectives (firms, networks, regions ...) have explanatory primacy is of course part of an old debate in economics, sociology and the philosophy of science and is often now dealt with under the heading of "methodological individualism" versus "methodological collectivism" (Hayek 1945, Popper 1957, Coleman 1964, Douglas 1986).

Further potential for conceptual differentiation relates to the forms, channels and mechanisms of knowledge exchange. As this exchange occurs through interaction, the structure of the interaction therefore influences the extent of knowledge diffusion (Gay and Dousset 2005). This coincides within the view that "spatialities and temporalities are not neutral frames, but constitutive elements of socioeconomic transformation" (Colletis-Wahl et al. 2008).

The cross-sectoral dimension of knowledge spillovers is also a source of contention in the literature. Following Marshall (1890) and Arrow (1962) knowledge is predominantly industryspecific. Knowledge spillovers may therefore arise between firms within the same industry. Jacobs (1969), on the other hand, mentioned the significant fact that knowledge may spill over between complementary rather than similar industries.

The significance of geographical agglomeration and networking is strongly determined by the particular sector (industry) and the leading technology. There seems to be a clear agreement in the recent literature about cross-sectional differences in agglomeration forces: As has been emphasized by Botazzi et al. (2001 and 2002) and also Gordon and McCann (2000), huge intersectoral differences in spatial agglomeration outcomes can be identified.

Following Gordon and McCann (2000) agglomeration economies appear particularly relevant in "scale-intensive sectors" hinting at the forms of hierarchical agglomeration discussed above - and in "supplier-dominated sectors". Conversely, they appear the least relevant in "science-based" sectors. The importance of agglomeration depends on the prevailing sectoral and technological pattern.

The following argumentation takes up two approaches to differentiating typologies and focuses on the different dimensions of agglomeration and 
clustering viewed as helpful guidelines in the discussion of the network observed in Styria and in answering the key-questions of the empirical analysis. An attempt is also made to combine and differentiate the "agglomeration" approach with the additional insights mentioned above.

Gordon and McCann (2000, 15) define and discuss three theoretical approaches for industrial clustering which reflect different (more or less idealized) perspectives on agglomeration: The model of pure agglomeration, the industrialcomplex model and the social-network model.

- The phenomenon of economies of agglomeration as an intrinsic motive for clustering, in the sense of spatial concentration of economic activity, is attributed more or less exclusively to the traditional idea of Marshallian industrial districts. Following in the footsteps of Thünen in the field of locational economics, and Smith's idea of division of labour, the model of pure agglomeration which in the tradition of Marshall (1890) is based on a local pool of specialized labour, on the increased local provision of nontraded input specific to an industry, and on technological spill-overs - may contribute to an "evolving localized environment of learning" (Gordon and McCann 2000, 517). The Marshallian approach was quickly developed and extended by Hoover (1948) by distinguishing between localization economies and urbanization economies. Following Marshalls (1890) positive externalities of agglomerations are defined by regional non-traded inputs, knowledge and information spill-overs, and a local pool of skilled labour. From the perspective of knowledge flows and learning processes favoured by agglomeration, such externalities occur more or less unheard of and unseen. Knowledge exchange and learning occurs unconsciously via transfer of human or material resources. The most important point seems to be that the approach is not bound to the idea of direct supplyrelationships among the bulk of actors involved. Following the traditional idea of Marshallian industrial districts, interaction is primarily led by the needs of industrial production.

- A second group of approaches pooled by Gordon and McCann (2000, 517) under the term of industrial complex models systematically tries to justify spatial concentration by the quantification and minimization of spatial transaction costs (reflecting of the origins of the approach, primarily transportation costs). The industrial complex model is associated with cumulative learning from sources inside the industry, non-transferable experience, the role of leading firms and power asymmetries (Iammarino and McCann 2005). Although the implicit concealment of (unplanned) economies of agglomeration didn't mean that they were not relevant. Attention shifted nevertheless to innovation as an interactive process involving the sharing and the exchange of different forms of knowledge between actors (Lawson and Lorenz 1999) knowledge and competence as developed interactively and within subgroups of a (regional) economy (Freeman 1979, Lundvall 2002). The critique here has been concerned with the question of whether this interaction is an outcome of (neoclassical) rational behaviour or the result of a more 'associative-relational' mode of organization, or what has been termed 'associative governance', leading to the creation of clubs, forums, consortia and other institutional schemes of partnership (Cooke 1998; Cooke and Morgan 1998). There are elements of knowledge sharing in the sense that adopting the perspective of specific clusters represents a quasi-monopoly for the internalization of the benefits of innovation created within (more or less) "closed club".

- The social-network model as the third type - relying on trust and social embeddedness as the dominant link between the cluster firms (and therefore not on deliberate economic decisions based on the minimization of different transaction costs) - also favours the exchange of knowledge. However, such exchange is here based on strong interpersonal relationships that transcend firm boundaries and allow for diverse forms of knowledge sharing. Following Iammarino and McCann (2005) traditional and recent approaches of social networks may be differentiated. The traditional approach corresponds to the 'Marshall-stimulated' industrial districts where knowledge is mainly codified and oriented to process innovation transferred by personal contacts and social and political lobbying. While in the traditional approach the network seems to be based on geographical proximity rooted in historical experience, the new approach of social networks seems to be 
based on relational and organizational proximity. The links between actors are then all the stronger the more they are based on elements of social embeddedness: norms, sets of common assumptions, habits formed by culture, history, and of course (but not necessarily) spatial proximity. They form social capital that favours the explicit and implicit sharing of knowledge. New physical technologies are not just there, innovations do not just happen, but need social technologies as pathways to coordinate human action.

As Iammarino and McCann (2005) mention, much of the discussion in the literature is based on ideal types, whereas in reality all spatial clusters and industrial agglomerations will contain more or fewer of the above characteristics. Furthermore, clusters may mutate from one typology to another. From another perspective this is also outlined by Rychen and Zimmermann (2008, 768): The concept of cluster "usually considered as a spatial concentration of industrial and technological activities" has to be enriched. "It is more important to understand how and why firms build links and how the structure of links will give sense to the colocation of actors." It is therefore important to incorporate the dimension of collaboration, the basic conception of firms is a "network-driven economic strategy built on collaboration among the participants" (Reid et al. 2008, 2).

The following section is dedicated to interpreting the case network investigated in the light of the approaches discussed above. The suppositions that context, typology and significance of geographical agglomerations and embedded networks change, seems to be reflected in the case of the machinery sector in the region of Styria.

\section{The empirical analysis - a qualitative context oriented approach based on social network analysis}

The empirical analysis starts with an analysis of relevant regional data and expert interviews and then continues with a case study analysis of the relations of engineering firms in Styria.

\subsection{Interaction in the observed network}

Network analysis is a well established method in the social sciences. Recently, the method has also been applied to the analysis of production clusters (Krätke 2002), innovative activity and knowledge exchange (Giuliani 2005), and alliance networks (Gay and Dousset 2005) or R\&D networks.

Social network analysis is a helpful tool for discussing the structure of networks since it allows the mapping and measuring of the relationships (communication and transaction) between different actors, i.e., the existence, context and portfolio of relations between actors in a regional network. It is a method for revealing relations between different actors. Such relations are phenomena that cannot be reduced to the properties of individual actors or firms themselves and thus need to be interpreted as properties of systems than of individual actors.

\subsection{The empirical database}

The present network analysis is based on an empirical sample of firms identified by a snowballing method of sampling in cluster and network investigation. This corresponds with the relational approach and is developed by means of the references to actors as revealed by previous respondents (Frank 1979, Scott 2000).

Our starting point was a large system supplier in the automobile sector located in the region of Styria/Austria. The snowball method produced firms belonging to different sub-sectors of the manufacturing sector and related supply-chain and innovation-strategies. Starting with the initial firm, a sample was developed. Following a citation path of regional suppliers (production or commercialization of goods and services) and of regional partners in the field of research and development (cooperative R\&D and related activities and exchange). In this way the database for the subsequent network analysis was extended to 23 firms, of which 18 are producers (with different positions in the supply-chain such as system-suppliers, component suppliers and tollmanufacturers). The remaining 5 are technical business services. Additionally nine R\&D institutions (universities, co-operative R\&D institutions) are included. The information and data collected are based on extensive qualitative interviews and supported by a quantitative survey concerning specific data.

\subsection{Indicators of interaction}

Qualitative indicators revealing individual strategies of innovation are helpful discussing individual strategies and their aggregation at the level of networks. They are selectively used here to find - via network analysis - the structural features of the network of 32 actors. The selected indicators of the relations cultivated by the organizations cover three dimensions of interaction: direct delivery relations, R\&D, and technological innovation in a competitive and a pre-competitive context.

(DELIV): The firms were questioned concerning direct delivery relations (goods or services) to clients, suppliers or partners (in the case of synergetic product bundles)..The direct delivery 
of goods and services is not reduced to the material dimension since it also covers questions of innovation in information management or capacityextending investments.

(PRE-COMP): The dimension of interaction in the context of pre-competitive $R \& D$ was also analysed. Pre-competitive Research and Development aim to extend the product spectrum, as well as introduce new processes and alternative materials. Pre-competitive research includes fundamental research, which is an activity designed to broaden scientific and technical knowledge not necessarily linked to industrial or commercial objectives, as well as industrial research, i.e. research aimed at developing or improving new or existing products, processes or services in so far as it is also not directly connected with a client tender, offer or an existing business relation.

(COMP): Competitive Research and Development and innovation processes are short and medium term oriented and mostly associated with direct expectations of return or with a direct tender or offer etc. in contrast to pre-competitive $\mathrm{R} \& \mathrm{D}$ which is long-term oriented.

\subsection{Structure of the network and network density}

Following the socio-centric approach, the density of a network is given by the ratio of relations realized to the total number of potentially maximum possible relations. We dichotomized the relations in that we only differentiated between existence and non-existence of a relation between two actors [0; 1], and therefore disregarded the intensity of the relations (in our case the frequency of interaction) surveyed. This enabled us to avoid the problems typically associated with the measurement of the intensity of evaluated graphs (Scott 2000). Network density yields information on the general structure of the network as a whole.

One of the core features of an actor identified in network analysis is its centrality. Using the concept of centrality (in different forms) we gain insights into the specific features of the interaction of the actors in the network and their specific position and/or embeddedness in the network. While density focuses on the properties and general structure of the network as a whole, centrality tries to capture the position of individual actors or groups of actors within the network. This is again based on the relations revealed by the actors, where the relations are valued ordinally in terms of frequency of interaction. The potential centrality of an actor is determined by a broad range of industry or sectorspecific factors (Cohen et al. 2000), by capacity and individual motivation (Bayona et al. 2001, Theter 2002). A high centrality is positively associated with multiple possibilities for receiving and generating knowledge.
Keeping in mind that interregional and international relations exist and may be of major priority, e.g. direct delivery relations the analysis below focuses on regional interaction. Table 1 presents the density measure for the three dimensions of relations between the actors.

\begin{tabular}{|l|l|r|}
\hline \multicolumn{2}{|l|}{ Relational dimensions } & Density \\
\hline (DELIV) & $\begin{array}{l}\text { direct delivery } \\
\text { relations }\end{array}$ & 0.068 \\
\hline $\begin{array}{l}\text { (PRE- } \\
\text { COMP) }\end{array}$ & $\begin{array}{l}\text { interaction in } \\
\text { the context of } \\
\text { pre- } \\
\text { competitive } \\
\text { R\&D }\end{array}$ & 0.143 \\
\hline (COMP) & $\begin{array}{l}\text { interaction in } \\
\text { the context of } \\
\text { competitive } \\
\text { R\&D and }\end{array}$ & 0.074 \\
\hline
\end{tabular}

\section{Table 1: Density of the observed dimensions of networking}

Direct delivery relations have the weakest density. Although the datasets have been dichotomized and therefore relations with a very low frequency of interaction have been "up-graded" the density of the network of direct delivery relations is lower than the density of knowledge intensive innovationrelated interaction. Regional input-output relations were reduced in order to focus attention more on international markets.

While competitive R\&D and innovation processes, especially in the case of domestic system suppliers, are partially similar in density to direct delivery relations, the regional density of the network in pre-competitive $\mathrm{R} \& \mathrm{D}$ is much higher. $R \& D$ institutions are of negligible significance in respect of direct delivery relations, the network is based to a considerable degree on relations with cooperative $R \& D$ institutions.

The relational data can be used to provide a graphical representation of the transaction network for the organizations observed. While network diagrams offer a traditional and basic methodology for formalizing network analysis, and are a very helpful mean of interpretation and discussion, clarity suffers greatly as the number of actors observed increases.

A quite useful method of graphical representation which is implemented in most software packages follows the approach of the Kamada and Kawai (1989) spring embedding algorithm. This is employed below. 
gives an overview of all relations recorded and combines the three dimensions discussed above. It also takes into account the valuation of the relations in terms of frequency of interaction. The shape of

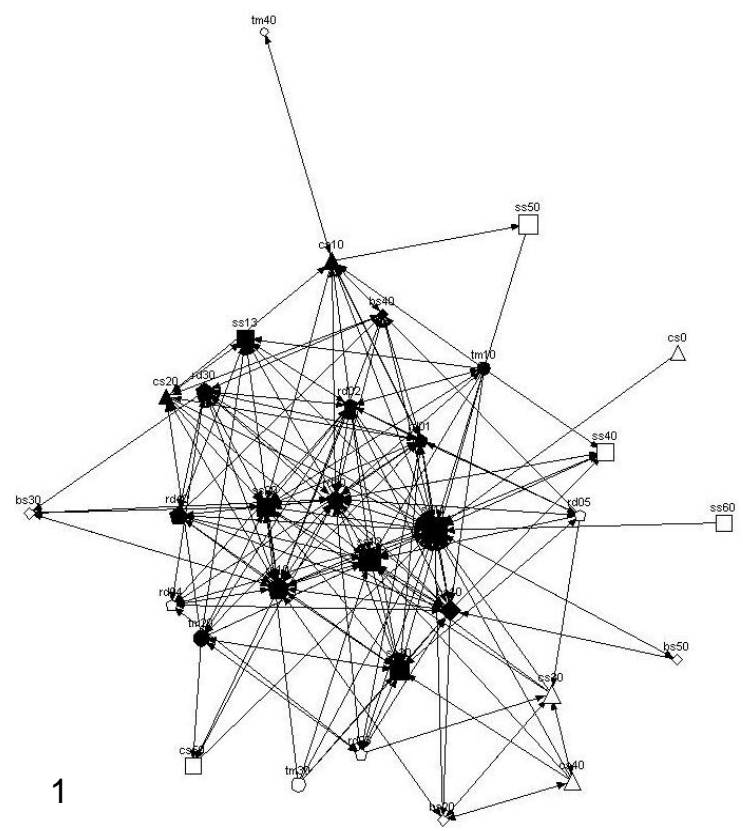

actors (nodes) corresponds with the different types of organizations. The size of the nodes corresponds with the size of the organisation, and the length of lines corresponds with the distance between the actors observed.

Figure 1: Network of firms and knowledge generating institutions

Figure 2: Legend

A further interesting dimension of network analysis is 'coreness', which follows basically the idea of core and periphery. Here we use the concept of the $\mathrm{k}$-core (Seidman 1983, Scott 2000). A k-core is a sub-graph in which each actor is adjacent to at least $\mathrm{k}$ other actors in the sub-graph. That is, for all nodes in the sub-graph minimum the number of the actors' direct relations within the sub-graph is $\mathrm{k}$ (in our case eight). K-core analysis complements the measurement of density, since the latter is not able to reflect structural features of the network. The kcore is an area of relatively high cohesion. As can be seen at first glance, we can differentiate between those actors in the core of the network (coloured black) and those actors more or less on the periphery of the network (coloured white). The diagram reveals the high density of the realized relations calculated in the previous paragraphs. In the $\mathrm{k}$-core of the diagram we find a group of institutions that seem to interact multilaterally. In the "core" of the network we find R\&D institutions, large system suppliers and toll manufacturers (surface-treatment, heating etc.) which maintain multiple but weak relations with a broad range of regional clients.

\subsection{Spotting a leading firm in the network}

Here we focus on a specific firm, ss 20 in the total network. This is a highly specialized manufacturer who measures equipment for science and industry. Their success is based on the direct application and transfer of scientific knowledge gained in the measurement of physical or chemical phenomena.

The firm is highly vertically integrated and is embedded in smaller network following niche strategies. The partners of the firm in direct delivery (component and toll-manufacturers) and its partners in competitive and pre-competitive research and development (key clients, highly specialized business services, universities) are not identical.

On the delivery side, the observed firm interacts with component suppliers in the field of die casting, spray casting, plastics processing, electronics, sheet metal forming, and manufacturing of high performance glasses.

Originally, the firm was a pure converter, producer and specialist in marketing. This division of labour has changed since the 1980s. A wellestablished cooperative base allows access to university partners and to an independent research laboratory which provides exclusive science driven $\mathrm{R} \& \mathrm{D}$. The firm enjoys a relatively high in-degree 
centrality in respect to direct deliveries. The outdegree centrality of the firm in the region, with respect to deliveries, is low owing to the high export intensity.

A high share of the turnover is reinvested in $R \& D$ activities, $10 \%$ for intramural $R \& D$ and an additional $10 \%$ of the turnover for external R\&D. The degree centralities in respect to $R \& D$ (precompetitive and competitive) are higher than for the average of the leading firms in the network. The core competences of the firm are based on combining and applying findings from basic research, in precision engineering and electronics.

While radical innovations and market novelties mostly emanated from R\&D or client-partners, incremental improvements are promoted by internal R\&D. R\&D and production and marketing of new products are concentrated within the region.

As the firm is not located in the core of vehicle manufacturing but in the interface with other sectors such as manufacturing of plastic products or measurement techniques it has a relatively high value for betweenness centrality. The findings for this specific firm serve to strengthen the thesis that firms acting in market niches demanding highly specialized cooperation tend to work for long-term cooperation.

\subsection{The historical background and changing role of geographic agglomeration in the medium-technology sector in Styria}

The majority of the observed firms have been in the region for more than 10 years. The current situation and recent developments cannot therefore be adequately analysed without considering the historical background and structural change of the regional industry over the last few decades.

In the late 1960s and 1970s the networks in the medium-technology sector were dominated by large state-owned firms that were highly vertically integrated and had lost their headquarter functions to the city of Vienna. While supply-side linkages to the region still existed agglomeration took a very limited traditional form. In most cases, planning, R\&D and marketing/distribution functions, i.e. those functions responsible for the monitoring of markets and technology, had been lost. Clearly observable lock-in effects had let to agglomeration becoming a mere by-product of path-dependence with none of the advantages of agglomeration mentioned by Botazzi et al. (2001).

Against the background of a history of outward dependence and nationalized standardized mass production the traditional indicators used to measure the strength of social networks had become weak. According to Iammarino and McCann (2005) social networks exhibit the following characteristics: Knowledge is largely codified and mature and mainly oriented to process innovation, transmitted essentially by way of personal contacts; there is extensive social and political lobbying, backward and forward linkages. As far as social networks still existed (e.g. in the machinery and the automobile sector) they became a fruitful base for the restructuring in the 1990 s.

During the developments of the last few decades the typology of agglomeration and the role of networks have changed considerably. Many large firms were re-privatized and downsized at the end of the 1980s. Firms thus needed to learn to collaborate and develop their potential for innovation as a strategic resource. This entailed abrupt and long overdue changes from a Fordist to a more flexible mode of production.

A massive structural change took place, beginning in the 1990s, especially in sectors related to steel production such as mechanical engineering and the automotive industry. High degrees of diversification and broad unspecified clients were replaced by a focus on market niches and technological specialization, while higher lot sizes enabled higher cross-functional integration and raised flexibility by leaving a scope for automation. Technological upgrading (including the introduction of quality and measuring standards) opened doors to new clients. This was accompanied by extending their responsibilities for tool making and sourcing capabilities and also by shifting the responsibility for quality and price from clients to suppliers. Innovations in these sectors were influenced by applications of specialized knowledge in the field of materials, tooling and processing techniques, or by the need to solve very specific problems in the machinery sector. On the supply side, hierarchical, spatially localized relations were developed. These have been formed around elite $\mathrm{R} \& \mathrm{D}$-intensive export-oriented large firms.

\subsection{Human resources and the regional labour market}

A typical characteristic of agglomerations, in the sense of the model of pure agglomeration mentioned by Gordon and McCann (2005), following Botazzi et al. (2001) - namely, a more or less common labour market pool - was not observed. For the investigated component supplier firms (here in more or less rural and isolated areas) it is still the case that they operate with reference to very local labour markets, binding traditions and a low mobility of employees. Small and medium sized supplier firms exhibit family-based traditional structures, sometimes over generations. Concerning the qualification structure, there are deep differences between Original Equipment Manufacturers or system-suppliers with R\&D units on the one hand, and basic technology providers, 
extended work-benches or third party subcontractors on the other hand.

This is true for both lower as well as high skilled workers, where employee turnover is normally a more or less excepted instrument of knowledge transfer and networking among firms. Because of the immobility of the local labour force and the restricted capacity of the regional labour market most of these firms were able to retain keypersonnel and competences and the regionally integrative potential of their personnel. Yet, by the same token, this implies only little mobility of qualified personnel coming from Europe (for language reasons, predominately from Germany). Also due to official restrictions labour inflow from the new EU member countries remains limited.

\subsection{Discriminative capabilities and heterogeneous strategies in the case of $R \& D$ and Innovation}

As already mentioned, leading firms do not play a dominant role on the demand side. The broad range of material input-output linkages is directed outward and direct material linkages on the regional level areweak. In fact the opposite seems to be true. In the case of large firms, agglomeration phenomena based on knowledge complementarities (Botazzi et al. 2001) seem to be clearly evident.

The R\&D capacities of the observed firms were highly varied. Nearly half of the investigated firms (mostly SMEs) do not employ permanent R\&D staff. The leading firms have intensified $R \& D$ activities and formal co-operation with knowledgegenerating institutions since the mid of the 1990s. Especially in the case of pre-competitive R\&D cooperative publicly supported projects or participation in cooperative $R \& D$ institutions has gained an increasing role as a policy measure during the last decade.

In respect to knowledge-driven activities, elements of agglomeration phenomena based on knowledge complementarities were observed (following Botazzi et al. 2001). These were also in line with the exclusivity characteristics suggested by the industrial complex model (Gordon and McCann 2005). The large $R \& D$ intensive firms observed here, constantly seek forms of regional pre-competitive $R \& D$ cooperation. This may result in the formation of "Clubs" (Gordon and McCann 2005, Cooke 2000) of closer interaction especially in respect of $\mathrm{R} \& \mathrm{D}$, or in some cases cooperative R\&D institutions. While material input-output linkages are spreading widely and are outward oriented the R\&D-oriented sphere is concentrated on the local context. This to a large extent supported by intensive direct and indirect social interaction (informal exchange, contacts in the local technical community). During the past few years, in terms of innovation firms already active in $R \& D$ have undergone a shift from being demand pull driven (responding to market demands) to technology push driven (firms have become proactive in their search for new technologies and USPs). This has increased the motivation to be integrated in the regional (technical) science community. The main spheres of economies of agglomeration have shifted considerably during the last few decades. The newly identified research 'Clubs' in publicly supported R\&D-projects are able to utilize economies of agglomeration primarily concentrated in the field of $R \& D$ and science.

As long as natural spill-overs are high and competitive conflicts are manageable (e.g. in the case of material sciences) larger firms accept weaker partners and smaller firms and are willing to integrate them into their activities. Low spill-overs and a higher market orientation favour more restrained, sometimes exclusive behaviour from the stronger party. This corresponds with the findings in the literature for partner selection in $\mathrm{R} \& \mathrm{D}$ cooperations (Atallah 2005).

This form of agglomeration, partially taking place beyond formal networks, also corresponds with the idea of a new type of social network mentioned by Iammarino and McCann (2005). Firms engaging in cooperative pre-competitive $\mathrm{R} \& \mathrm{D}$ and knowledge generation appeared to seek suitable equal partners.

The qualitative interviews strengthened the notion that firms attempt to generate a portfolio of cooperative partners which consciously combines specialization and flexibility. In terms of knowledge generation and exchange, the geographic dimension is relevant as long as the actors are able to utilize knowledge potential. While larger firms with noticeable R\&D-capacities are able to utilize international contacts in research and development activities, smaller low- or mediumtech firms stick to the region and to their regional partners. Smaller firms are confronted with a selfreinforcing combination of low $R \& D$ capability on the one hand, and limited market demand on the other.

In agreement with the concept of absorptive capacity, it was found that firms with low $R \& D$ and innovation potential (mainly component suppliers, where innovation is predominately directed by investment) found it difficult to build up and retain adequate relations with knowledge generating organizations. The medium-tech component suppliers observed here, proved to be unable to maintain continuous relationships with knowledge generating institutions. They were not capable of defining, setting up and managing relevant projects. These low and medium-tech firms did, however, partially utilize opportunities to establish long-term contacts with individual public or semi-public R\&D-institutions (dealing with basic technologies 
such as material sciences). They also tried to gain from possible spill-overs from appropriate events or informal inquiries. Here we found that, in direct delivery and in competitive and pre-competitive research and development (as far as existent) is not identical.

In addition, two interesting long-term partnerships between small knowledge intensive technical business services and large systems and component suppliers were observed, in the network analysed here. They were based on long-term trust and informal exchange.

\section{$4 \quad$ Final remarks}

The roles of clusters, networks and geographical agglomeration are subject to considerable coevolution. Different approaches concerning forms, channels and mechanisms of knowledge exchange offer different conclusions with respect to the significance of geographical agglomeration in knowledge exchange.

In the case study analysis different dimensions of interaction can be observed. There are networking-dimensions of material, supply-oriented transactions and networking-dimensions of knowledge sharing. The first belongs to the process of division of labour dealing with the exchange of goods and services, the second with knowledge. The main differences reside in the form of interaction and in the impact of interaction. The spheres of physical interaction (subcontracting relations) differ considerably from the spheres of knowledge intensive and R\&D-driven interactions. They are different in respect to actors involved, spatial extension, and significance of geographic agglomeration.

The observed network is, in its regional dimension, dominated by knowledge intensive relations. The qualitative evidence gathered by numerous in-depth interviews reveals that the highest number of interactions was reached in precompetitive R\&D knowledge exchange and that immaterial dimensions dominate the material ones. The (industrial) firms do have extensive supplier relations but only to a very limited extent within the region and within the network. There is no automatic parallelism of interactions. This does not necessarily exclude automatic spill-over of knowledge connected with supplier relations, but it does emphasize that higher intensities of knowledge exchange, as indicated by the revealed forms of interaction are actively chosen and not a mere byproduct. Knowledge oriented relations within the network are to a large degree regionally concentrated. Proximity per se is not sufficient to generate knowledge between firms. The diffusion of knowledge within clusters is highly selective and depends strongly on the position of firms within networks and their absorptive capacity. Especially in pre-competitive research, local universities and cooperative $R \& D$ institutions play a dominating role and assume gatekeeper functions. Firms with a relatively high $R \& D$ capacity also take up such a role, thus indicating the necessity of a welldeveloped internal knowledge base. The dominating role of the newly founded cooperative R\&D institutions (competence centres) can be taken as an indication that this kind of network relation is rather new and that the pattern of interaction has a temporary character and depends of the existence of specific kinds of knowledge generating institutions. In the Styrian case, the main dimensions of economies of agglomeration have changed considerably during the last few decades. The portfolio of interactions and the meaning of agglomeration for the observed firms cannot be reduced to specific dimensions taken as a given or not. To this extent that they are determined by firm capabilities and firm behaviour, not all dimensions of agglomeration and thus economies of agglomeration are accessible for all agents. While small and medium sized firms partially gain from economies of agglomeration in the field of basic technologies such as material sciences or tool making, large firms concentrated pre-competitive research in the region in order to gain from economies of agglomeration in the field of science and R\&D.

These agglomeration effects still seem to be concentrated around certain insider 'clubs'. A considerable share of the firms investigated is not able to participate and gain from economies of agglomeration. While there is a long tradition of pro-active cluster and network promotion in Styria sectorial diversity (i.e. a low critical mass of actors) and relatively low absorption capacity serve to hamper the potential gains from economies of agglomeration for a considerable number of SMEs.

\section{References}

Amin A. and Thrift N. 1995. Globalisation, institutional 'thickness' and the local economy. In Healy P., Cameron S., Davoudi A., Managing Cities: The New urban Context. Wiley, Chichester.

Arrow K. 1962. The economic implications of learning-by-doing. Review of Economic Studies 29: 155-173.

Atallah G. 2005. Partner Selection in $R \& D$ Cooperation. CIRANO - Centre interuniversitaire de recherche en analyse des organisations, Montréal.

Bayona C., Garcia-Marco T., Huerta E. 2001. Firms' motivations for cooperative R\&D: an empirical analysis of Spanish firms. Research Policy 30: 1289-1307. 
Belussi F. 2006. In search of a theory of spatial clustering: agglomeration versus active clustering. Clusters and Regional Development. Routledge, London.

Botazzi G., Dosi G., Fagiolo G. 2001. On the Ubiquitous Nature of the Agglomeration Economies and their Diverse Determinants: Some Notes, LEM Laboratory of Economics and Management Working Paper 10. St. Anna School of Advanced Studies, Pisa.

Botazzi G., Dosi G., Fagiolo G. 2002. Mapping Sectoral Patterns of Technological Accumulation into the Geography of Corporate Locations. A Simple Model and Some Promising Evidence. LEM Laboratory of Economics and Management Working Paper 21. St. Anna School of Advanced Studies, Pisa.

Cohen W.M. and Levinthal D.A. 1989. Innovation and Learning. The Two Faces of R\&D. The Economic Journal 99: 569-596.

Cohen W.M., Nelson R.R., Walsh J.P. 2000. Protecting Their Intellectual Assets. Working Paper. National Bureau of Economic Research.

Coleman J.S. 1964. Collective décisions. Sociological Inquiry 34: 166-81.

Colletis-Wahl K., Corpataux J., Crevoisier O., Kebir L., Pecqueur B., Peytache-Gadeau V. 2008. The territorial economy: a general approach in order to understand and deal with globalization. Aranguren Querejeta M.J., Networks, Governance and Economic Development. Edward Elgar, Cheltenham.

Cooke P. 2000. Structural competitiveness and learning régions. Enterprise \& Innovation Management Studies 1.

Cooke P. 1998. Regional innovation systems. The role of governances in a globalized world. UCL Press, London.

Cooke P. and Morgan K. 1998. The Associational Economy: Firms, Regions and Innovation. Oxford.

Dixon R.J. and Thirlwall A.P. 1975. A Model of Regional Growth-Rate differences on Kaldorian Lines. Oxford Economic Papers 27: 201-14.

Dosi G. and Malerba F. 1996. Organization and Strategy in the Evolution of the Enterprise. MacMillan, London.
Douglas M. 1986. How Institutes Think. Syracuse University Press. Syracuse, NY.

Felin T. and Foss N.J. 2006. Individuals and Organizations: Thoughts on a MicroFoundations Project for Strategic Management and Organizational Analysis. DRUID Working Paper: 06-1.

Foss N.J. 1999. The Use of Knowledge in Firms. JITE - Journal of Institutional and Theoretical Economics 155: 458-486.

Frank O. 1979. Estimation of population totals by use of snowball samples. Perspectives on Social Network Research, 319-347. New York.

Freeman L.C. 1979. Centrality in social networks: Conceptual clarification. Social Networks 1: 215-239.

Gay B. and Dousset B. 2005. Innovation and network structural dynamics: Study of the alliance network of a major sector of the biotechnology industry. Research Policy 34 (10): 1457-75.

Giuliani E. 2005. The structure of clusters. Knowledge and Networks: Uneven and Selective, not Pervasive and Collective. DRUID Working Paper 05-11.

Gordon I.R. and McCann P. 2005. Innovation, agglomeration, and regional development. Journal of Economic Geography 5 (5): 52343.

Gordon I.R. and P. McCann 2000. Industrial clusters: complexes, agglomeration and/or social networks? Urban Studies 37: 513-532.

Granovetter, M. 1994. Business Groups. The Handbook of Economic Sociology, 453-475. Princeton.

Grant, R.M. 1996. Towards a Knowledge-Based Theory of the Firm. Strategic Management Journal 17: 109-122.

Hartmann C. 2006. Die Lernfähigkeit von Clustern. Eine theoretische und empirische Betrachtung. Leykam, Graz.

Hayek F.A. 1945. The use of knowledge in society. University Press. Chicago.

Hoover E. 1948. Location of Economic Activity. MacGraw Hill Book Company, Columbus, Ohio.

Iammarino S. and McCann P. 2005. Firm Location and Technology: Stylised Constructs and Illusory Policies. Paper presented at the 4th European Meeting on Applied Evolutionary Economics (EMAEE). Utrecht, 18-25 Mai. 
Jacobs J. 1969. The Economy of Cities. Random House. New York.

Kaldor N. 1972. The Irrelevance of Equilibrium Economics. The Economic Journal 82.

Kamada T. and Kawai S. 1989. An Algorithm for Drawing General Undirected Graphs. American Sociological Review 33: 499-517.

Krätke S. 2002. Network Analysis of Production Clusters: The Potsdam/Babelsberg Film Industry as an Example. European Planning Studies 10: 27-54.

Lawson C. and Lorenz E. 1999. Collective learning, tacit knowledge and regional innovative capacity. Regional Studies 33: 305-317.

Mariotti F. and Delbridge R. 2001. Managing Portfolios of Ties in Inter-firm Networks. Paper presented at the Nelson and Winter Conference. Aalborg, Denmark.

Marshall A. 1890. Principles of Economics. Macmillan, London.

Maskell P. and Malmberg A. 1999. Localized learning and industrial competitiveness. Cambridge Journal of Economics 23: 167185.

Myrdal G. 1957. Economic Theory and UnderDeveloped Regions. Gerald Duckworth \& Co., London.

Nonaka I., Toyama R. and Nagata A. 2000. A Firm as a Knowledge-Creating Entity: A New Perspective on the Theory of the Firm. Industrial and Corporate Change 9: 1-20.

Powell W.W. and Smith-Doerr L. 1994. Networks and Economic Life. The Handbook of Economic Sociology, 386-402. Princeton.

Popper K.R. 1957. Science: Conjectures and Refutations. The Growth of Scientific Knowledge, 33-65. Routledge, London.

Reid N., Smith B.W. and Carroll M.C. 2008. Cluster Regions. A Social Network Perspective. Economic Development Quarterly.

Rychen F. and Zimmermann J.B. 2008. Clusters in the global knowledge based economy: Knowledge gatekeepers and temporary proximity. Regional Studies 42(6).

Scott J. 2000. Social Network Analysis - A Handbook. Sage, London.

Seidman S.B. 1983. Network Structure and Minimum Degree. Social Networks 5: 269287.

Storper M. 1995. The resurgence of regional economies ten years later: the region as a nexus of untraded interdependencies.
European Urban \& Regional Studies 3: 191221.

Storper M. 1997. The regional world: territorial development in a global economy. Guildford Press, London.

Theter B.S. 2002. Who cooperates for innovation, and why: An empirical analysis. Research Policy 31: 947-67.

Weber A. 1929. Theory of the Location of Industries. The University of Chicago Press. Chicago. 